\title{
Una mirada al performance de Orlan desde una lectura del $\mathrm{CsO}^{*}$
}

\author{
Steven Silva Salazar*
}

Recibido: 26 de marzo de 2015 - Revisado: 23 de junio de 2015 -

Aprobado: 14 de agosto de 2015

La obra de arte puede ser mirada, y es la perspectiva desde la que se construye este texto, como una forma de pensamiento, y en este contexto, como una forma de producción de la filosofía. Se trata de ver cómo el mismo objeto artístico se convierte en un fenómeno de interpretación de la vida, y así ofrece respuestas vitales a la misma cotidianidad, tanto del artista/creador como del espectador. El ejercicio surge con ocasión de la visita de la reconocida y polémica artista francesa Orlan al Museo de Arte Moderno de Bogotá, de forma que este artículo tiene como propósito hacer un acercamiento estético y filosófico al performance de la artista, desde sus inicios hasta sus últimas obras fotográficas, a través de la perspectiva de cuerpo sin órganos; concepto filosófico utilizado por G. Deleuze y F. Guattari (2000).

Palabras clave: Orlan, arte carnal, performance, cuerpo sin órganos.

* Artículo de reflexión

** Licenciado en Filosofía y Letras por la Universidad Santo Tomás. Magister en Filosofía, Pontificia Universidad Javeriana. Profesor del departamento de Humanidades, Universidad Santo Tomás, Colombia. Dirección postal: carrera 9 \# 51-11, Bogotá (Colombia), correo electrónico: stivensilvasalazar@usantotomas.edu.co 


\title{
A look at the performance of Orlan from the reading of the BwO*
}

\author{
Steven Silva Salazar*
}

The artwork can be looked at, and it is the perspective from which this text is constructed, as a form of thinking, and in this context, as a form of philosophical production. It is about seeing the same artistic object become a phenomenon of interpretation of life, and thus offers vital answers to the same everyday life, both of the artist/creator and of the spectator. The exercise arises from the visit of the renowned and controversial French artist Orlan to the museum of Modern Art of Bogota, so this article has as purpose to make an aesthetical and philosophical approach to the artist's performance, since her beginning to her last photographic works, through the perspective of body without organs; philosophical concept used by G. Deleuze and F. Guattari (2000).

Keywords: Orlan, carnal art, performance, body without organs.

* Reflection article.

** Bachelor in Philosophy and Letters from the University of Santo Tomás, Master in Philosophy Pontifical Xaverian University. Professor of Humanities, University, of Santo Tomás, Colombia. Postal address: carrera 9 \# 51-11, Bogotá (Colombia), e-mail: stivensilvasalazar@usantotomas.edu.co 


\section{Un regard au performance de Orlan à partir d'une lecture du CsO*}

Steven Silva Salazar*

L'œuvre d'art peut se regarder, est c' est depuis cette perspective que se construit cet article, comme une manière de penser, et dans ce contexte, comme une forme de production de la philosophie. Il s'agit de voir comment le même objet artistique se transforme en un phénomène $d$ 'interprétation de la vie, et offre ainsi des réponses vitales au quotidien même, aussi bien celui de l'artiste/ créateur comme celui du spectateur. L'exercice naît à l'occasion de la visite de la polémique et reconnue artiste française Orlan au Musée de 1'Art Moderne de Bogota, de cette façon cet article a pour but de réaliser une approche esthétique et philosophique au performance de l'artiste, depuis ses débuts jusqu'à ses dernières œuvres photographiques, à travers de la perspective du corps sans organes; concept philosophique utilisé par G. Deleuze y F. Guattari (2000).

Mots clés: Orlan, art charnel, performance, corps sans organes

* $\quad$ Article de réflexion.

** Titulaire d'une maîtrise en Philosophie et Lettres de I'Université Santo Tomás. Magister en Philosophie, Université Javeriana. Professeur du département de Sciences Humaines, Université Santo Tomás, Colombie. Adresse postale: carrera 9 \# 51-11, Bogota (Colombie), e-mail: stivensilvasalazar@usantotomas.edu.co. 
Al abordar el arte desde una perspectiva filosófica se pueden tomar dos posibles caminos, sin pretender que estos sean absolutos o totalizantes: en primer lugar, tomar la obra de arte como un objeto de reflexión filosófica; aplicar sobre él conceptos estéticos, antropológicos o políticos y disertar sobre la manera en que estos dan cuenta de aquella producción humana. Un segundo camino es entender el arte como una forma de pensamiento, y en este contexto, como una forma de producción de la filosofía; se trata de ver cómo el mismo objeto artístico se convierte en un fenómeno de interpretación de la vida, y así, ofrece respuestas vitales a la misma cotidianidad, tanto del artista/creador como del espectador. Este ensayo tomará la segunda vía, analizando el performance de la artista francesa Orlan, intentado desde él responder a la pregunta que Deleuze y Guattari (2000) plantean en su obra Mil Mesetas, a saber: ¿cómo hacerse un cuerpo sin órganos?

Dicen Deleuze y Guattari (2000) que el cuerpo sin órganos (CSO) “de ningún modo es una noción, es un concepto, más bien es una práctica, un conjunto de prácticas" (p. 156). Afirman que no se trata de una receta logarítmica o unos pasos determinados y establecidos que se pueden seguir para llegar a construirse uno, es más bien, un modo personal que se debe forjar cada individuo para conseguir estar en él; se trata entonces de experimentar, tanto en el terreno biológico, político, estético y vital, aun cuando las sociedades capitalistas tengan formas preestablecidas del mismo cuerpo e impidan o censuren dicha experimentación: “os impedirán experimentar en vuestro rincón" (Deleuze y Guatari, 2010, p. 156).

Esta concepción de la vida no es una idea abstracta y general. [Deleuze y Guattari necesitan] mostrarla en carne viva y, por lo tanto, debe ser considerada con relación al cuerpo, al cuerpo tal y como es tratado, experimentado y pensado en el arte (Chirrola, s.f.).

Lo que se pretende es hacer una afirmación de la experimentación sobre el cuerpo -o con el cuerpo-, y en el caso del arte, nos es posible pensar que la cuestión se podría aclarar más fácilmente, pues, al hacer tal cosa sobre su propia posesión (que sería el cuerpo ${ }^{1}$ ) se estaría ejerciendo una práctica creadora que no se limita a interpretar el mundo o su propia personalidad a través de patrones establecidos, sino de crear nuevas realidades y escenarios, que permiten abrir la experiencia humana a un mundo de intensidades, velocidades y matices, ante los cuales, el cuerpo debe abrirse rompiendo su subjetividad orgánica, organizada y establecida, para permitir la emergencia de nuevas realidades. "Encontrad vuestro cuerpo sin órganos, sed capaces de hacerlo, es una cuestión de vida o muerte, de juventud o de vejez, de tristeza o de alegría. Todo se juega a ese nivel" (Deleuze y Guattari, 2010, p. 157). Así lo afirman Deleuze y Guattari, es una actitud vital. Romper estructuras capitalistas de interpretación sobre el cuerpo y la realidad; renunciar al psicoanálisis en su búsqueda exhaustiva del yo interior que deja vestigios de su pasado para entender su

1 Sin querer decir o intentar resolver la pregunta: “¿somos un cuerpo o tenemos un cuerpo?”, esto nos llevaría a un análisis fenomenológico, el cual no estamos intentando resolver en este ensayo. 
presente; para optar por un esquizoanálisis, en donde no importa el yo porque lo que se pretende es llegar a tales niveles de abstracción, en donde solo quede lo necesario para la recepción de intensidades, fuerzas, velocidades y cambios de intensidades en dirección encontradas, es decir, cambios de gradientes. Así entonces, el CSO es algo que se construye o que está por construirse a través de un ejercicio de experimentación, el cual debe propender hacia la apertura del cuerpo a un sinnúmero de conexiones con potencias de todo tipo (conexiones a las $n$ potencias). Entonces, podemos hablar de CSO cuando se ha suprimido todo: el conjunto de significancias y de subjetivaciones.

Con este panorama, debemos ser enfáticos en la distinción de dos elementos: "no hay que confundir exactamente lo que pasa sobre el CSO y la manera de hacerse uno" (Deleuze y Guattari, 2010, p. 158). Ante tal afirmación, podemos hacer la siguiente disertación: supongamos un plan de consistencia, con estratos y territorios bien definidos, controlados por sistemas capitalistas pequeñoburgueses, en donde la vida se presenta de la forma más molar posible, es decir, más cotidiana y rutinaria; donde podemos decir que no hay devenires sino futuro. Para hacerse un cuerpo sin órganos que se oponga a toda la organización establecida, es necesario trazar líneas de fuga o flujos de desterritorialización, los cuales se fijan en un plan de inmanencia que se ubica detrás de los otros planes coagulados en series definidas. Dichas líneas de fuga, flujos de desterritorialización que nos pueden reterritorializar en un nuevo plan, no son los mismos para todos, no funcionan de la misma manera. He ahí la necesidad de la experimentación. No se trata de seguir caminos ya recorridos por otros, se trata de asumir esta actitud: "caminante no hay camino, se hace camino al andar". Como veremos más adelante, se trata de "caminar" con prudencia, de ser cauto en la experimentación para que la línea de fuga no se convierta en línea de muerte, pues, ya Deleuze (1995) nos ha dicho: "todo cuanto he escrito -al menos así lo espero- ha sido vitalista" (p. 228). Se trata pues, de afirmar la vida, no de llegar a la autodestrucción.

Llegamos pues a la respuesta a la pregunta inicial: ¿cómo hacerse un cuerpo sin órganos? Orlan ha encontrado su propia manera de experimentación, lo que ella llama L'art charnel:

Carnal Art is self-portraiture in the classical sense, but realised through the possibility of technology. It swings between defiguration and refiguration. Its inscription in the flesh is a function of our age. The body has become a "modified ready-made", no longer seen as the ideal it once represented; the body is not anymore this ideal ready-made it was satisfaying to sign (Orlan, s.f.).

En Orlan: Accouche d'elle - m'aime (figura 1), Orlan nos nuestra la manera en que su práctica de construcción en el terreno artístico tiene principio:

Le brasgauchereplié sur le visage, en position de méditation, et donne naissance à un corps inerte et androgyne, ni homme ni femme. Les deux visages regardent dans la même direction. Cet 
acte, qui ouvre la suite des poses nues dans l'atelier, constitue, symboliquement, la tentative de se donner naissance, de s'inventer une nouvelle identité (Orlan, 2004, p. 60).

Esta es pues la primera manera en que Orlan empieza a experimentar con su cuerpo, transgrediendo una primera ley establecida: hace un parto de sí mismo. La misma dirección en la que miran los dos cuerpos da pie a la adopción de un nuevo nombre, "un nombre de guerra": Orlan ha nacido para trasgredir y para hacer de su cuerpo un agenciamiento maquínico de protesta, a la sociedad capitalista y psicoanalítica ${ }^{2}$. Un esquizoanálisis de la sociedad será el que emprende Orlan para mostrar cómo el cuerpo puede ser otra cosa, elemento de protesta, tanto artístico como político.

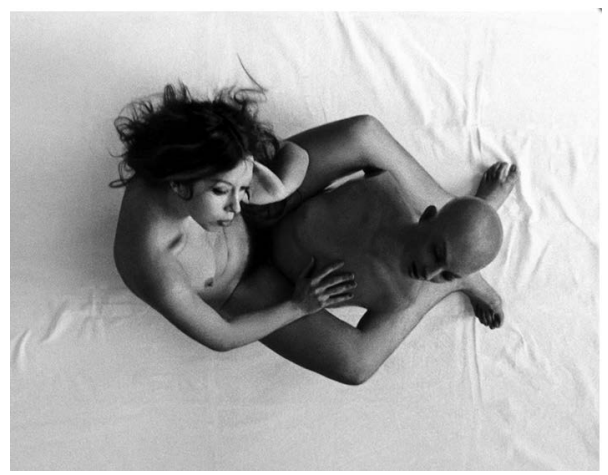

Figura 1. Orlan: Accouche d'elle-m'aime (1964). Orlan, 2014.

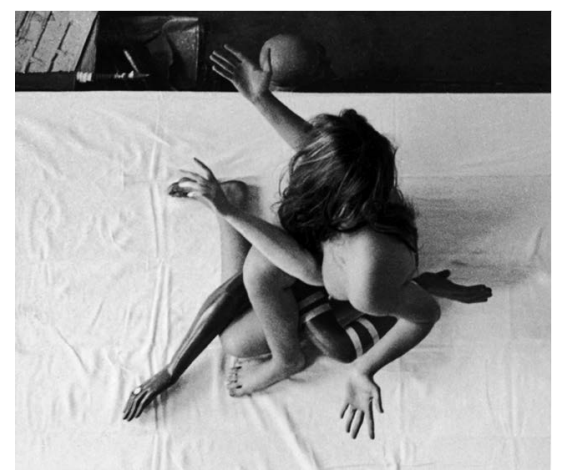

Figura 2. Orlan: Corps - sculpture no. 3, dit Shiva ou tentacules de bras multiples, (1964).

Decimos psicoanalítica en el sentido en que ella misma afirma: "[...] le choix de ce nom s'impose à la suite d'un séanse de psychanalyse (...) et comprend alors, après l'intervention (...) qui pointe l'anomalie, qu'elle ne sera plus jamais dan cet état de mort lent, et s'attribue une autre identité civil. Comme ce corps mort à qui elle donne naissance, le nom qu'elle choisit n'est ni féminin, ni masculin. II renferme la revendication d'Orlan de transgresser les tabous et d'être en marge des modèles de différenciations" (Orlan, 2004). 
Emprende la desfiguración del nuevo cuerpo nacido, re-figurándose a través de retorcimientos, haciendo la experiencia de las deformaciones por fuerzas invisibles que lo llevan a tomar distintos aspectos.

Orlan ha resucitado, ahora viene a intervenir con otra identidad, aquella que se ha desterritorializado, para intervenir y hacer la ruptura a las normas capitalistas, va a hacer del cuerpo una desfiguración para re-figurarse en un CsO que se prestará al paso de nuevas intensidades. Ya Deleuze y Guattari (2000) nos han descrito una serie de cuerpos que pueden ser principio de $\mathrm{CsO}$ : cuerpo hipocondríaco, cuerpo paranóico, cuerpo esquizofrénico, cuerpo drogado, cuerpo masoquista. Si vemos este último, por ejemplo, su iniciación se hace atar, coser, "inmovilizar para detener el ejercicio de los órganos" y luego latigar, herir, etc. Se trata aquí de una primera instancia; de neutralizar el organismo para poder pasar a una segunda fase, la cual consiste en hacer pasar algo a través. Orlan no se hace pegar o atar; más bien revolotea y retuerce su cuerpo como una escultura.

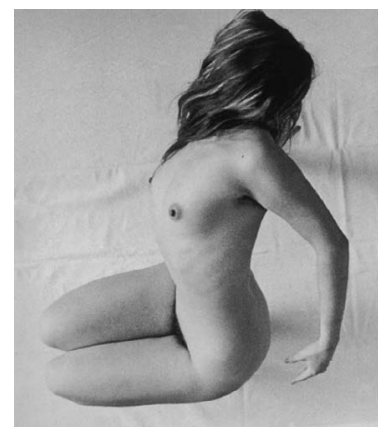

Figura 3. Orlan: Corps - sculpture sans visage et au bras droit, (1965).

Esta escultura (figura 3) quiere salir del cuadro, quiere una vida propia, hay un devenir-arte en ella, un bloque de devenir que convertirá a esta imagen en otra cosa y le impedirá volver a ser la que era. Prestará su cuerpo, no para enfrentarse a los órganos sino a la organización que hace los órganos.

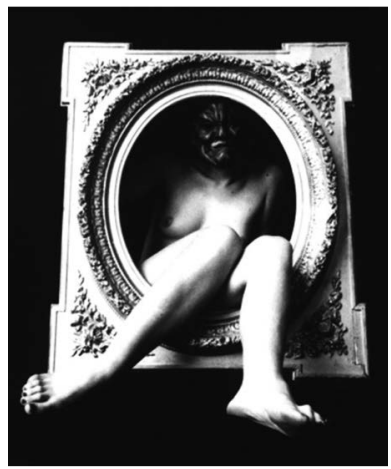

Figura 4. Orlan: Tentative pour sortir du cadre avec masque, (1965). 
En Orlan: Tentative pour sortir du cadre avec masque (figura 4) vemos cómo Orlan, el transgresor, toma su propia identidad y ya no solo es una obra de arte, es más que eso: es un cuerpo vivo, que viene a hacer resistencia.

Terminado su devenir/escultura, Orlan está lista para hacer fluir intensidades a través de ella, y así, hacer un CSO social y político, que se enfrenta a líneas molares y segmentos duros, para hacer sobre ellos una nueva experimentación, una nueva re-interpretación y construirse un nuevo mundo desarticulado. Puede emprender tal ruta, haciendo una serie de acciones en las calles, en espacios neutros.

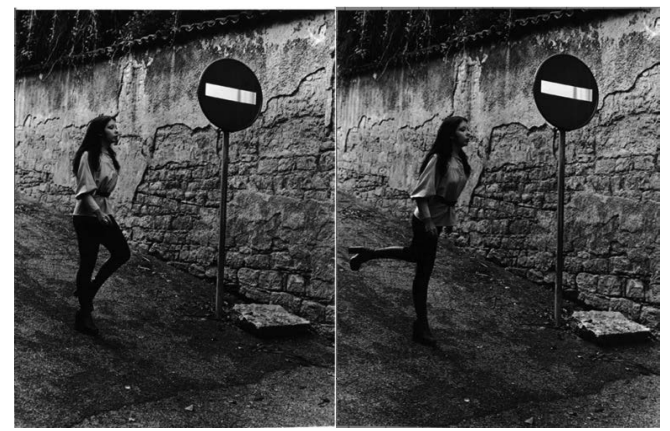

Figura 5. Orlan: Action Or-lent: les marches au ralenti dite au sens interdit.

Vemos cómo estas fotografías son el fiel testimonio de nuevas actitudes urbanas, en las cuales, lo primero que salta a la vista es una señal de prohibición. Lo que quiere mostrar Orlan es su deseo de transgredir, eliminar las prohibiciones. Asistimos, en pleno 1964 en Francia, a la idea según la cual "les marches publiques, on le sait, sont des instruments de revendication politiques, sociales et économique" (Orlan, 2004, p. 68.) Esta es una clara apropiación de un gesto contestatario, pues, aunque silenciosa y solitaria, afirma la denuncia y se inscribe en una nueva forma de vivir la calle, la cual, pretende tomársela, entendiendo que ningún gesto, elección o compromiso, son neutros. Este es el debut del cuerpo como instrumento de conocimiento con relación a otros en el espacio; Orlan se ha hecho algo público, y así, se abre a la intensidad que ella denominará la "mesuRage".

El ingenioso término es acuñado por la artista hacia 1974, con el cual quiere indicar una serie de acciones que se demarcan por la palabra rage (rabia), con la cual, Orlan quiere refutar toda las series de roles que la sociedad capitalista, las segmentariedades duras y los planos de consistencia propios de los microfascismos; quiere establecer y ponderar, formas de vida perfectamente delimitadas, estructuras urbanas y estéticas que definen la vida. Estos son los flujos de desterritorialización.

El concepto de Estado totalitario solo tiene valor a escala macropolítica para una segmentariedad dura y para un modo especial de 
totalización y de centralización. Pero el fascismo es inseparable de núcleos moleculares, que pululan y saltan de un punto a otro, en interacción, antes de resonar todos juntos en el Estado nacionalista. Fascismo rural y fascismo de ciudad o de barrio, joven fascismo y fascimo de excombatiente, fascismo de izquierda y de derecha, de pareja, de familia, de escuela o de despacho: cada fascismo se define por un microagujero negro central generalizado (Deleuze y Guattari, 2010, p. 34).

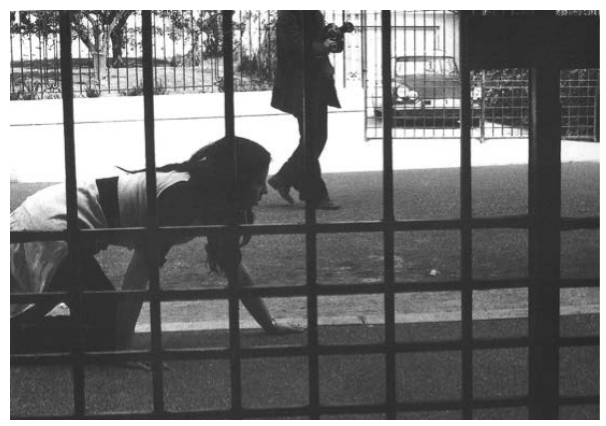

Figura 6. Orlan: MesuRage de la rue Chateaubriand à Nice.

Esas micropolíticas o microfascismos, molares o sobrecodificados, son los que determinar la vida, es lo que Orlan medirá (mesurage), pero en términos de rabia (mesuRage), de protesta, de resistencia (si se permite el término: de indignación) para hacer emerger un flujo que nos lleve a una vida no fascista. Adopta un aspecto simple y hasta salvaje; animal: se posa en cuatro patas y mide las calles.

À partir de cette marques au sol, elle répète le geste autant de fois que nécessaire pour prendre possesion de l'endroit désigné, à la manière d'un animal qui repére son territoire. Elle compte ensuite le nombre d'Orlan -corps- effectués et l'inscrit sur le constat (Orlan, 2004, p. 102).

Esta acción se emparenta al gesto de provocación, ante el espacio urbano y el lugar que este ocupa, o mejor aún, la manera determina de ser ocupado. Hay una nueva forma urbana que se mide en términos de rabia, para hacer fluir los cuantos en contravía.

“Si ce MesuRage s'incrit dans la suite de ceux évoqués précédemment, cette interventionà liège prend un caractère nettement plus politique" (Orlan, 2004, p. 108). El CsO en constante proceso de construcción y experimentación sobrepasa el terreno estético y crea flujos moleculares tanto políticos como sociales. La protesta, en términos de rage, llega a tal intervención, hasta desfragmentar su cuerpo, romper e intervenir en la organización de los órganos para poder hacer fluir de ahora en adelante solo intensidad, velocidades y fuerzas. 


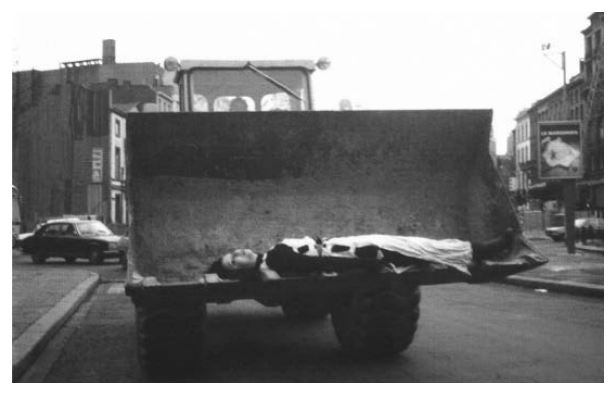

Figura 7. Orlan:MesuRage de la place Saint - Lambert à Liège.

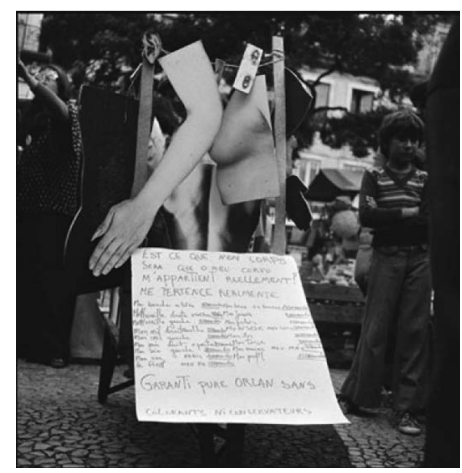

Figura 8. Orlan: se vendre sur le marchés en petits morceaux.

El resultado es el siguiente (figura 9): una apertura a toda clase de sensaciones e intensidades.

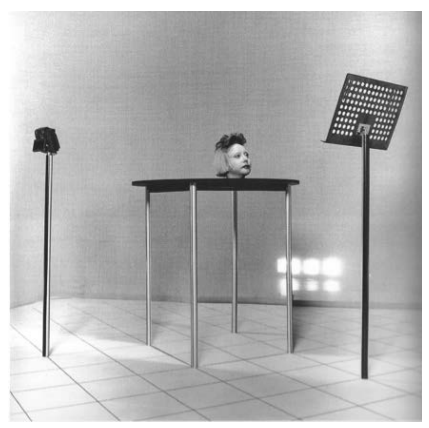

Figura 9. Orlan: Femme avec tête plan général et visage de trois quart no. 5.

Se ha logrado deshacer Orlan de los tres grandes estratos que impiden el CsO: el organismo, la significancia y la subjetivación. Ahora solo quedan articulaciones (las $n$ articulaciones), que pueden partir de intensidades $=0$; una materia no formada que hace pasar intensidades y las distribuye por lo que atraviesa. Si 
bien, dijimos con Deleuze y Guattari (2000) que es muy diferente, la manera de hacer un $\mathrm{CsO}$ y lo que pasa sobre él, vemos cómo se construyó, que ahora está dispuesto a intensidades. Fue necesaria la prudencia en la experimentación, en el proceso o la práctica que ha vaciado el cuerpo, para poder llenarlo de otra cosa y no dejarlo vacío, pues el vacío es la muerte. No se trata de las drogas (aunque muchas cosas pueden servir como drogas, o el embriagarse con agua), las cuales, dejan en el cuerpo solo ondas de frío que vacían otra posibilidad de experimentación, más drogas para más frío. El yonqui es un cuerpo vaciado en las ondas frigoríficas, es todo un teatro que busca el gran frío, esto es destrucción: muerte.

Hay varias maneras de fallar el CsO. Si bien, cuando no se produce nada, cuando no se logra hacer pasar intensidades es porque algo lo está bloqueando. El CsO es una constante oscilación entre los estratos de la superficie y los planes que lo liberan; no obstante, liberarlo de aquello que lo bloquea de forma violenta, es decir, destruir los estratos con violencia, "os habréis matado a vosotros mismos". Se trata es de instalarse en un estrato, experimentar las posibilidades que este ofrece para encontrar los movimientos de desestratificación; las posibles líneas de fuga. Asegurar la conjunción de flujos a través de la experimentación, un continuum de intensidades: tener siempre fragmentos de una nueva tierra.

Orlan se deshizo de su organismo, ya experimentó en los estratos y tiene los fragmentos de una nueva tierra. Ahora solo la atraviesan intensidades, lo que ella llama la Défiguration - refiguration. Self - hybridation, los movimientos de desestratificación que le llevaran a un devenir precolombino.

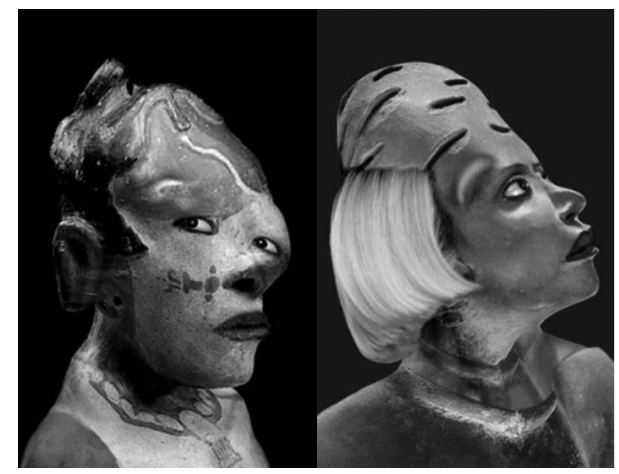

Figura 10. Orlan: Défiguration - refiguration. Self-hybridation précolombienne no. 1. Défiguration - refiguration. Self-hybridation précolombienne no. 2. Orlan. 


\section{Referencias}

Brigante, A. M. (2005). La relación del cuerpo con el arte: del cuerpo mecánico al cuerpo sin órganos. El cuerpo, fábrica del yo. Bogotá: Editorial Pontificia Universidad Javeriana

Chirrola, G. (s.f.). Vitalismo y cuerpo sin órganos en Gilles Deleuze. Recuperado de http://www.javeriana.edu.co/cuadrantephi/sumario/articulos22.htm

Chirrola, G. (2005). El paseo de Orlando. El cuerpo: una matriz de subjetivación y de des-subjetivación. El cuerpo, fábrica del yo. Bogotá: Editorial Pontificia Universidad Javeriana.

Deleuze, G. (1995). Conversaciones 1972-1990. Valencia: Pre-Textos.

Deleuze, G., y Guattari, F. (2000). Mil mesetas (capitalismo y esquizofrenia). Madrid: Pre-Textos.

Orlan. (s.f.). Manifesto of carnal art. Recuperado de http://www.orlan.net/texts/

Orlan. (2004). Orlan. Éditions Flammarion. París.

\section{Referencias iconográficas}

Orlan: Orlan accouche d'elle - m'aime. 81x76 Photographie noir et blanc, tirage unique. 1964.

Orlan: Corps - sculpture no. 3, dit Shiva ou tentacules de bras multiples. Photographie noir et blanc, tirage unique. 1964.

Orlan: Corps - sculpture sans visage et au bras droit. $81 \times 76 \mathrm{~cm}$, photographie noir et blac, tirage unique 1965 .

Orlan: Tentative Pour sortir du cadre avec masque. 6,5x10,5 cm. Photographie noir et blanc, tirage unique. Collection Fonds d'art contemporain, Paris. 1965.

Orlan: Action Or-lent: les marches au ralenti dite au sens interdit. Photographie noir et blac.

Orlan: MesuRage de la rue Chateaubriand à Nice, France. Photographie noir et blanc.

Orlan: MesuRage de la place Saint - Lambert à Liège, Belgieque. Photographie couleur. Prose de vue, Jean - Paul Lefret. 
Orlan: se vendre sur le marchés en petits morceaux. Performance réalisée à Caldas da Rainha. Photographie Blanc et noir.

Orlan: Femme avec tête plan général et visage de trois quart no $5.50 \times 60 \mathrm{~cm}$, photographie noir et blanc. 3 exemplaires. Prise de vue, Peter Sinclair.

Orlan: Défiguration - refiguration. Self - hybridation précolombienne no 1. 100x150 cm, cibachrome collé sur aluminium, cadre. Collection Jacques Kerchache.

Orlan: Défiguration - refiguration. Self - hybridation précolombienne no 2. 100x150 cm, cibachrome collé aluminium, cadre. Collections MNAM-CCI, centre George Pompidous, Paris. 